\title{
La Ley de Modernización para la Seguridad Alimentaria (FSMA): Controles Preventivos para los Alimentos de Animales ${ }^{1}$
}

\author{
Francisco Rivera, Chad Carr, y Jason M. Scheffler ${ }^{2}$
}

\section{Introducción}

La Ley de Modernización de la Seguridad Alimentaria (FSMA, por sus siglas en inglés) se convirtió en ley en enero de 2011 y se considera la reforma más amplia de las normas de seguridad alimentaria en 70 años. El impulso detrás de esta legislación incluyó algunos incidentes importantes, como un brote de Salmonella Typhimurium en la mantequilla de maní (crema de cacahuate) de la Peanut Corporation of America (CDC 2009), un brote de Listeria monocytogenes en los melones canteloupes (CDC 2011) y un retiro masivo de alimentos para mascotas debido a presencia de melamina (FDA 2016a). Primero, se establecieron las regulaciones sobre alimentos para el consumo humano luego con la contribución de la industria, la academia, y los consumidores y otras agencias, se modificaron para adaptarse mejor a la producción de alimentos para animales.

En Florida, estas nuevas regulaciones se aplican a las instalaciones que fabrican, procesan, empacan o almacenan alimentos o ingredientes para animales. La Administración de Alimentos y Medicamentos (FDA) usa el término "alimento." Sin embargo, "alimento" puede sustituirse según corresponda. Estas instalaciones pueden incluir fabricantes de alimentos para mascotas, procesadores, destiladores de etanol, fábricas de procesamiento, distribuidores y otros. El objetivo principal de estas regulaciones es garantizar alimentos seguros para los animales, las personas que manejan el alimento y las personas que consumen productos derivados de animales.

\section{¿Qué requiere la regla?}

Las instalaciones sujetas al reglamento deben establecer un plan la inocuidad de los alimentos. Este plan debe incluir un documento escrito que integre buenas practicas de manufactura (CGMPs; por sus siglas en inglés), un análisis de riesgos y prevención de controles (cuando sea necesario). CGMPs son prácticas utilizadas para garantizar la limpieza general de las instalaciones, la capacitación del personal y las operaciones adecuadas de la planta. Por ejemplo, procedimientos para el lavado de manos de los empleados, mantenimiento y limpieza de equipos y control de plagas. El análisis de riesgo identifica y evalúa la prevención de peligros conocidos o razonablemente riesgosos asociados con cada alimento producido, empacado o retenido que puedan causar enfermedades o lesiones a humanos o animales. Los peligros se clasifican como biológicos, químicos, o físicos. Incluyen, pero no están limitados a patógenos, deficiencias de nutrientes y fragmentos de metales. Las CGMPs pueden ayudar a manejar

1. Este documento, AN330-Span (the English version of this document is The Food Safety Modernization Act (FSMA) Preventive Controls for Animal Food (AN330)), es uno de una serie de publicaciones del Departamento de Ciencias Animales, Servicio de Extensión Cooperativa de la Florida, Instituto de Alimentos y Ciencias Agrícolas, Universidad de la Florida (UF/IFAS Extension). Fecha de primera publicación: febrero 2019. Visite nuestro sitio web EDIS en $<$ https://edis.ifas.ufl.edu>.

2. Francisco Rivera, small farms and alternative enterprises Extension agent, UF/IFAS Extension Hillsborough County; Chad Carr, associate professor; and Jason M. Scheffler, assistant professor, Department of Animal Sciences; UF/IFAS Extension, Gainesville, FL 32611. 
estos peligros. Sin embargo, la instalación debe optar por implementar controles preventivos específicamente dirigidos a los riegos que puedan ocurrir con frecuencia y tengan probabilidad de repetirse. Estos controles deben incluir pasos específicos en los procesos, estableciendo controles de saneamiento o controles colocados en la cadena de suministro. Estos componentes básicos deben incluir procedimientos apropiados para el monitoreo, validación y registro de datos en las operaciones.

Además de los controles preventivos, existen otros tres componentes principales de la regla: programas de verificación de proveedores extranjeros, certificación para organizaciones privadas (terceros) y transporte sanitario. Luego de los controles preventivos, la verificación de proveedores extranjeros es la segunda preocupación inmediata, debido a que puede afectar un pequeño porcentaje de las instalaciones.

\section{¿Esta regla se aplica a mi instalación?}

La respuesta a esta pregunta depende de los detalles de su operación. Si su instalación ya está registrada en el FDA como una instalación nacional o extranjera que fabrica, procesa, empaca o almacena alimentos como se define en 21 CFR 1.227 (https://www.gpo.gov/fdsys/granule/CFR2012-title21-vol1/CFR-2012-title21-vol1-sec1-227/contentdetail.html) (Richardson et al. 2015), lo más probable es que sí se aplique.

Hay ciertas excepciones. Por ejemplo, si una fábrica de alimentos es utilizada exclusivamente para fabricar alimentos para los animales en la granja donde se encuentra, puede cumplir con la definición de una granja de producción primaria y estar exenta de la regla. Sin embargo, si su granja produce alimento para el consumo de su propio ganado, pero se entrega a agricultores contratados, es posible que tenga que aplicarse, debido a que la administración que supervisa el ganado es diferente. Si su instalación produce ingredientes de alimentos para animales como un subproducto de la producción de alimentos humanos (como por ejemplo, la pulpa de cítricos de un fabricante de jugo de naranja), es posible que ya esté cubierto por las CGMPs para la producción de alimentos humanos. En este caso, la instalación tiene la opción de implementar un plan separado para la alimentación animal o mantenerlo bajo el plan de alimentación humana. Existen otras situaciones que pueden resultar en la exención de toda o parte de la regla. Sin embargo, es importante revisar los detalles de su instalación y determinar si cumple con los criterios establecidos en la ley. Tenga en cuenta que instituir CGMPs es una buena práctica, incluso si su instalación está exenta.

\section{¿Cuándo mi establecimiento debe cumplir con los requisitos de ley?}

La FDA ha establecido una fecha límite para cumplir con diferentes aspectos de la ley (Tabla 1). Las grandes empresas tienen un plazo más corto que las operaciones pequeñas o muy pequeñas. Para todas las empresas, la fecha límite para el establecimiento de CGMPs es un año antes de la fecha límite para el establecimiento de análisis de peligros y controles basados en el riesgo.

\section{¿Quién es responsable de escribir un plan de seguridad alimentaria en mis instalaciones?}

Un individuo calificado en controles preventivos (PCQI; por sus siglas en inglés) es responsable de desarrollar el plan de seguridad alimentaria. Esta persona debe tener la instrucción, la experiencia o ambas para desarrollar e implementar controles preventivos basados en el riesgo. Es una buena práctica contar con un equipo de seguridad alimentaria que incorpore personal de todas las instalaciones involucradas en el desarrollo y mantenimiento del plan de seguridad alimentaria. Esto ayuda a garantizar la integridad del plan de seguridad alimentaria, así como familiarizarse con el plan general entre el personal de toda la instalación. El propietario, operador o agente a cargo es responsable del programa de seguridad alimentaria y debe asegurarse de que el PCQI y el equipo de seguridad alimentaria estén seleccionados adecuadamente.

\section{¿Qué experiencia y educación es necesaria para ser un individuo calificado en controles preventivos?}

Existen múltiples maneras para obtener la calificación para ser un PCQI. La Alianza de Controles Preventivos de Seguridad Alimentaria (FSPCA; por sus siglas en inglés) ha trabajado con la FDA para desarrollar un plan de estudios estandarizado como una forma de cumplir con los requisitos. Este curso está diseñado para enseñar los conceptos necesarios para diseñar un plan de seguridad alimentaria. Puede haber varios programas de adiestramiento equivalentes. También se puede adquirir la experiencia en el área mientras se trabaja con un plan de seguridad alimentaria de 
controles preventivos basado en el riesgo. Esta experiencia debe documentarse como justificación de que no se necesita un programa de capacitación.

\section{Llevamos a cabo buenas prácticas en nuestra operación: ¿Comenzamos de nuevo?}

No, sin embargo las prácticas actuales deben revisarse para determinar cómo se articulan en un plan de seguridad alimentaria y si se necesitan hacer modificaciones. Por ejemplo, los fabricantes de alimentos que producen alimentos medicados ya deben cumplir con las CGMPs para alimentos medicados (9 CFR 225). En la mayoría de los casos, estas CGMPs establecidas se ajustarán a un plan de seguridad alimentaria con poca o ninguna modificación.

\section{¿Un plan de seguridad alimentaria es un evento que solo se escribe}

\section{una vez?}

No. Se espera que el plan de seguridad alimentaria sea tratado como un "documento vivo." Debe revisarse y evaluarse siempre que el producto o el proceso cambie, se produzca un brote de una enfermedad transmitida por los alimentos en un producto similar, o se hagan las medidas correctivas necesarias. El plan de seguridad debe revisarse al menos cada tres años. Un plan de seguridad adecuado es posible que no necesite cambios. Por lo tanto, la revisión del plan se debe documentar y tener disponible para cualquier representante del FDA.

\section{Para más información visite los siguientes enlaces en línea}

FDA (requisitos clave para los controles preventivos para la alimentación animal): http://www.fda.gov/downloads/ Food/GuidanceRegulation/FSMA/UCM461884.pdf

FDA (FSMA; información general sobre alimentación animal): http://www.fda.gov/AnimalVeterinary/Products/ AnimalFoodFeeds/ucm347941.htm

UF/IFAS Department of Animal Sciences (FSMA): http://animal.ifas.ufl.edu/FSMA/index.shtml
Food Safety Preventive Controls Alliance (FSPCA): https://www.ifsh.iit.edu/fspca

Southern Center for FSMA Training: http://sc.ifas.ufl.edu/

\section{Referencias}

21 CFR \$1.227. Accessed on September 14, 2016.

9 CFR \$225. Accessed on September 14, 2016.

CDC. 2009. "Multistate outbreak of Salmonella Typhimurium infections linked to peanut butter, 2008-2009." Accessed on August 29, 2016. http://www.cdc.gov/salmonella/2009/peanut-butter-2008-2009.html

CDC. 2011. "Multistate outbreak of listeriosis linked to whole cantaloupes from Jensen Farms, Colorado." Accessed on August 29, 2016. http://www.cdc.gov/listeria/outbreaks/ cantaloupes-jensen-farms/index.html

FDA. 2016a. "Melamine pet food recall of 2007." Accessed on August 29, 2016. http://www.fda.gov/AnimalVeterinary/ SafetyHealth/RecallsWithdrawals/ucm129575.htm

FDA. 2016b. "FSMA final rule for preventive controls for animal food." Accessed on August 29, 2016. http://www.fda. gov/Food/GuidanceRegulation/FSMA/ucm366510.htm

Richardson, S., R. Goodrich Schneider, M. A. Ritenour, M. D. Danyluk, and K. R. Schneider. 2015. The Food Safety Modernization Act and the FDA Facility Registration Program. FSHN13-07. Gainesville: University of Florida Institute of Food and Agricultural Sciences. http://www. edis.ifas.ufl.edu/fs231 
Tabla 1.

\begin{tabular}{|l|l|l|}
\hline \multicolumn{1}{|c|}{ Tamaño del negocio } & \multicolumn{1}{|c|}{$\begin{array}{c}\text { Subparte B } \\
\text { Buenas prácticas de manufactura } \\
\text { actuales }\end{array}$} & $\begin{array}{c}\text { Subparte C } \\
\text { Controles preventivos basados en el } \\
\text { Análisis de amenazas y los riesgos }\end{array}$ \\
\hline Otros & 19 de septiembre de 2016 & 18 de septiembre de 2017 \\
\hline Pequeños negocios (<500ETC) & 18 de septiembre de 2017 & 17 de septiembre de 2018 \\
\hline Pequeños negocios (<2.5 millones/año) & 17 de septiembre de 2018 & 17 de septiembre de 2019 \\
\hline ETC=Equivalentes a empleados a tiempo completo (FDA 2016b) & \\
\hline
\end{tabular}

